\title{
A Review of Drop-foot Corrective Surgery
}

\author{
GILLIAN HALL \\ All Africa Leprosy and Rehabilitation Training Centre, Addis Ababa, Ethiopia
}

\begin{abstract}
The late results of 65 tendon-transfer operations for drop-foot are reviewed, especially in relation to the various surgical techniques employed. It is concluded that from the patient's point of view range of movement was more important than gait. For young patients with good pre-operative mobility, single-tendon transfers were preferable, but for patients with inverted, deformed or stiff feet, where range of movement was less important than stability, the two-tendon Carayon techniques could be preferable.
\end{abstract}

\section{Introduction}

In this report 65 tendon transfer operations, which were performed on 54 patients to correct drop-feet due to leprosy, have been reviewed. All the operations were performed at the All Africa Leprosy and Rehabilitation Training Centre (ALERT) in Ethiopia between 1968 and 1976. The cases have been divided in to 5 groups according to the date of surgery, the procedure used and the surgeon who performed the operation.

Most cases had been assessed prior to surgery in the physiotherapy department and had been taught isolation exercises for the tendon or tendons to be transferred. They attended the department again after surgery and were taught isolation and co-ordination of the transferred tendon in its new site and were trained to use it when walking.

Patients were usually asked to return to the department for review of the surgery after 6 months, but this did not always happen. Most cases were reviewed by the surgeon or physiotherapist or both when they happened to return to the hospital for some other reason.

\section{Measurements}

These were taken using a foot protractor with the patient sitting with his knee bent. The angle between the posterior aspect of the lower leg and the sole of the foot was measured taking the neutral position between dorsiflexion and plantarflexion as $90^{\circ}$. Thus decreasing figures indicate increased dorsiflexion and increasing figures increased plantarflexion.

Received for publication 17 February, 1977. 


\section{Gait}

Where this is recorded it has been assessed as follows:

Good. The patient walks naturally. There is no difference in gait between the operated foot and a normal foot the other side.

Fair. The patient walks well. However, there is an obvious difference between the operated foot and a normal foot the other side. He may use the transfer quite well when he thinks about it, but tends to drag his foot when observed unnoticed.

Poor. The patient still tends to drag his foot, although there is some improvement in the position of the foot.

Bad. The operation has not improved the patients' gait or the position of the foot at all.

\section{Group A}

This small group consisted of 3 patients one of whom had had bilateral transfers. The operations were performed between 1969-1971 by one surgeon. He used Tibialis Posterior which he transferred anteriorly through the interosseous membrane and sutured to the tendon of Extensor Hallucis Longus and ligaments of the intercuneiform joints on the dorsum of the foot. The patients were seen for review between 5 and 7 years after surgery. Measurements taken at discharged and review averaged as follows:

\begin{tabular}{lcc}
\hline & Discharge & Review 5-7 years \\
\hline Active dorsiflexion & 82.5 & 87.5 \\
Resting position & 91.2 & 100 \\
Active plantarflexion & 100.7 & 108.7 \\
$\begin{array}{l}\text { Range of active dorsiflexion from the resting } \\
\text { position (designated range A) }\end{array}$ & 8.7 & 12.5 \\
Range of active dorsiflexion from active & & 21.2 \\
\hline plantarflexion (designated range B) & 18.2 & \\
\hline
\end{tabular}

\section{OBSERVATIONS}

Three of the feet ( 2 patients) had a good gait with straight stable feet and no ulcers. In the other case the gait was bad because the transfer had stretched out $20^{\circ}$ since discharge to a resting angle of $110^{\circ}$.

\section{Group B}

Of this group of 10 patients, 2 had had bilateral transfers. One was performed in 1968 and reviewed 6 years later, the rest were done in 1972 and 1973 and reviewed between 3-4 years later. The 3 surgeons concerned used Tibialis Posterior which they transferred anteriorly around the tibia and divided into 2 slips which were inserted into the tendons of Extensor Hallucis Longus and Extensor Digitorum Longus at the level of the tarsus. In some notes it was also recorded that a stitch was made between the transferred tendon and the Extensor Retinaculum. Measurements taken at discharge and at review averaged as follows: 


\begin{tabular}{lcc}
\hline & Discharge & Review 3-6 years \\
\hline Active dorsiflexion & 74.5 & 85.9 \\
Resting position & 84.3 & 94.8 \\
Active plantarflexion & 94 & 103.3 \\
Active range A & 9.7 & 8.9 \\
Active range B & 19.5 & 17.4 \\
\hline
\end{tabular}

\section{OBSERVATIONS}

\section{Gait}

This was assessed as described above. The resting angles and range of movement A of each group were between the figures indicated.

\begin{tabular}{lcc}
\hline \multicolumn{1}{c}{ Gait } & Resting angles & Range A \\
\hline Good 7 cases & $85-95$ & $5-26$ \\
Fair 4 cases & $100-102$ & $2-8$ \\
Poor 1 case & 110 & 8 \\
\hline
\end{tabular}

\section{Recovery}

In 2 cases there was recovery of Tibilais Anterior. One of these cases had a weak muscle preoperatively. At review he had a very high range of dorsiflexion but no ulcers. In the other case the recovered Tibialis Anterior caused inversion during the active phase of gait but no ulcers.

\section{Stability}

One other case had inversion causing an unstable foot postoperatively but the foot was mobile and there were no ulcers.

\section{Ulcers}

Two cases had toe-tip ulcers despite an apparently good gait. One case had severe multiple ulcers but was an unco-operative patient who would not wear prescribed shoes.

\section{Group C}

This was a group of 20 patients of whom 3 had had bilateral transfers. They were performed during 1974 and 1975 by 2 surgeons. The operation was a Tibialis Posterior transfer using the same technique as described above for group B. In cases with an inadequate passive range of dorsiflexion the tendo achilles was lengthened usually by a simple percutaneous approach. Of these 23 operations, 16 were reviewed between 3-12 months after surgery and 18 between 1-2 $\frac{1}{2}$ years after surgery. Nine cases were reviewed during both periods. Measurements recorded at discharge and review were as follows:

\begin{tabular}{|c|c|c|c|}
\hline & Discharge & Review 3-12 months & Review $1-2 \frac{1}{2}$ yr \\
\hline Active dorsiflexion & 83 & 88.5 & 87.2 \\
\hline Resting position & 92.3 & 96.9 & 98.3 \\
\hline Active plantarflexion & 98.5 & 104.2 & 107.2 \\
\hline Active range $\mathrm{A}$ & 9.3 & 8.4 & 11.1 \\
\hline Active range $B$ & 15.5 & 15.7 & 20 \\
\hline
\end{tabular}




\section{OBSERVATIONS}

1. Gait

Gait was assessed and the corresponding angles were as follows:

\begin{tabular}{|c|c|c|}
\hline Gait & Resting angles & Range A \\
\hline Good 3 cases & $83-90$ & $4-13$ \\
\hline Fair 12 cases & $90-110$ & $5-20$ \\
\hline Poor 7 cases & $95-110$ & $5-17$ \\
\hline $\mathrm{Bad} \quad 3$ cases & $105-115$ & $5-9$ \\
\hline
\end{tabular}

\section{Stability}

Of these 23 feet, 15 had complete nerve lesions, 2 had weak peronei, one had an active extensor hallucis and the rest had incomplete records. Postoperatively there were 6 cases of feet inverting, 5 of the complete lesions plus the one with an active extensor hallucis. In at least 3 of these cases inversion was present preoperatively but records were inadequate in the other 3 cases. Two of these cases had lateral border ulcers and stiffness, one had a lateral heel ulcer and stiffness. Two had no ulcers but the feet were very unstable in walking; the last caused no real problem. The inversion was noticed immediately after operation and in 4 cases an attempt was made to correct it by reoperating to tighten the lateral slip. It was not successful. In another case an attempt at correction was made later by transferring the Flexor Digitorum Longus tendon through the interosseous membrane and suturing it laterally into the tendon of extensor digitorum longus. This did not significantly improve the deformity either.

\section{Ulcers}

Twenty-two of the feet had evidence of preoperative tibial nerve damage with records of anaesthesia, dryness, cracks or ulcers. Only one case definitely had good sensation preoperatively. Fourteen of the cases had had ulcers preoperatively and of these 8 recurred after surgery. Four others had developed ulcers after surgery which were not recorded beforehand.

\section{Group D}

Between April and July, 1975 a series of operations were performed using a "Modified Carayon" technique. Of these 6 patients were seen for review between 4-12 months after surgery. One patient had had a bilateral transfer. The surgeon used 2 tendons. Tibialis Posterior was transferred circumtibially to the dorsum of the foot where it was sutured into the tendon of Tibialis Anterior. Flexor Digitorum Longus was transferred through the interosseous membrane and sutured to both the tendons of Extensor Hallucic Longus and Extensor Digitorum Longus in the lower leg. Measurements recorded at discharge and review were as follows:

\begin{tabular}{lcc}
\hline & Discharge & Review 4-12 months \\
\hline Active dorsiflexion & 83.9 & 86.1 \\
Resting position & 88.9 & 95.1 \\
Active plantarflexion & 91.7 & 98.3 \\
Active range A & 5 & 9 \\
Active range B & 7.9 & 12.2 \\
\hline
\end{tabular}


Gait was assessed and the corresponding angles were as follows:

\begin{tabular}{lcc}
\hline \multicolumn{1}{c}{ Gait } & Resting angles & Range A \\
\hline Good 1 case & 92 & 11 \\
Fair 4 cases & $90-95$ & $4-13$ \\
Poor 2 cases & $100-104$ & $5-15$ \\
\hline
\end{tabular}

\section{Group E}

From April, 1975 until May, 1976, 41 operations were performed using Professor Carayon's two-tendon technique. Tibialis Posterior and Flexor Digitorum Communis were both brought anterior through the interosseous membrane. They were sutured to the tendons of Tibialis Anterior and Extensor Hallucis Longus plus Extensor Digitorum Longus in the lower leg. Lengthening of the tendo achilles was also done in cases with an inadequate range of passive dorsiflexion. When done an open division was performed. In some cases a release of the tibial nerve was performed, at the same time to try to improve the circulation through the tibial artery and thus to the sole of the foot. Of these cases 15 patients, 4 of whom had had bilateral transfers, were seen for review between 3 and 11 months after surgery. Measurements recorded at discharge and review were as follows:

\begin{tabular}{lcc}
\hline & Discharge & Review 3-11 months \\
\hline Active dorsiflexion & 84.9 & 86.6 \\
Resting position & 87.6 & 92.4 \\
Active plantarflexion & 91 & 98 \\
Active range A & 2.7 & 5.8 \\
Active range B & 6.1 & 11 \\
\hline
\end{tabular}

\section{OBSERVATIONS}

1. Gait

This was assessed and the corresponding resting angle and range of movement $\mathrm{A}$ were as follows:

\begin{tabular}{llcc}
\hline & & Resting angles & Ranges of movement \\
\hline Good 6 cases & $75-95$ & $0-19$ \\
Fair 7 cases & $80-98$ & $0-9$ \\
Poor 4 cases & $95-114$ & $2-10$ \\
Bad 2 cases & $94-105$ & $0-2$ \\
\hline
\end{tabular}

\section{Range of movement}

At discharge the range of movement was very limited. Of these 19 feet, 9 had no active range from rest at discharge and one had no active range from full plantarflexion. After a few months the resting angle dropped and the plantarflexion increased thus the active range of dorsiflexion increased. At review 3 still had no active dorsiflexion from rest, but all cases had some active range from plantarflexion. The gait of the patients was often assessed as good or fair even though the patient had very little active range of movement. However the patients were much happier with the operation if they had a greater range. 


\section{Stability}

Preoperatively 17 of these feet had complete nerve lesions and 2 had weak peronei. Postoperatively some had slight inversion but in none had it caused any problems such as instability in gait or ulcers.

\section{Ulcers}

Preoperatively there was evidence of tibial nerve damage in 17 feet. In 2 cases good sensation was recorded. Ten patients had ulcers before surgery and 5 had recurrence of ulcers postoperatively.

\section{Recovery}

One patient had recovery of Tibialis Anterior. His records showed that he had had a complete lesion preoperatively.

\section{Summary}

\section{GROUP A. TPT INTEROSSEOUS ROUTE 1969-71}

The range of movement was good and had increased at review. The average resting position had dropped $9^{\circ}$ in the 5 years. The results seemed encouraging but too few cases were seen to make any judgement.

\section{GROUP B. TPT CIRCUMTIBIAL ROUTE 1968-1972/73}

There was a good range of active dorsiflexion both from rest and from full plantarflexion. This range did not alter much between discharge and review. The angles of maximum dorsiflexion and the resting angles had dropped by about $10^{\circ}$ at review. However this did not afford the overall good results as assessed by gait. Only one case was inverting.

\section{GROUP C. TPT CIRCUMTIBIAL 1973-75}

There was a good active range of dorsiflexion which increased slightly after $2 \frac{1}{2}$ years. Active dorsiflexion dropped by $5^{\circ}$ during the first year but then remained constant. The resting position dropped $4^{\circ}$ during the first year and another $2^{\circ}$ in the next year.

Of these cases, 6 had bad inversion postoperatively. An attempt to correct this by tightening the lateral slip was not successful.

\section{GROUP D. MODIFIED CARAYON TECHNIQUE 1975}

The range of movement was not as great as in the above 3 groups. During the first 6 months the resting position dropped by about $5^{\circ}$ and the range of movement increased about $4^{\circ}$. No cases were recorded with inversion.

\section{GROUP E. CARAYON TECHNIQUE 1975/76}

The range of active dorsiflexion was very poor at discharge. It increased during the first 6 months. The maximal dorsiflexion position was on the whole maintained and the resting position had dropped $5^{\circ}$. In none of the cases were there problems with inversion.

Further study at a later stage needs to be done for the Carayon Technique to provide a fair comparison with the other methods. 
A comparative summary of angles at discharge and review

\begin{tabular}{llccccc}
\hline & & Dorsiflex. & Rest. & Plantarflex. & \multicolumn{2}{c}{ Active range } \\
\hline \multirow{2}{*}{ Group A } & Discharge & 82.5 & 91.2 & 100.7 & 8.7 & 18.2 \\
& Review 6 yr & 87.5 & 100 & 108.7 & 12.5 & 21.2 \\
Group B & Discharge & 74.5 & 84.3 & 94 & 9.7 & 19.5 \\
& Review 4 yr & 85.9 & 94.8 & 103.3 & 8.9 & 17.4 \\
Group C & Discharge & 83 & 92.3 & 98.5 & 9.3 & 15.5 \\
& Review 6 mth & 88.5 & 96.9 & 104.2 & 8.4 & 15.7 \\
& Review 2 yr & 87.2 & 98.3 & 107.2 & 11.1 & 20 \\
Group D & Discharge & 83.9 & 88.9 & 91.7 & 5 & 7.9 \\
& Review 6 mth & 86.1 & 95.1 & 98.3 & 9 & 12.2 \\
Group E & Discharge & 84.9 & 87.6 & 91 & 2.7 & 6.1 \\
& Review 6 mth & 86.6 & 92.4 & 98 & 5.8 & 11 \\
\hline
\end{tabular}

\section{Conclusions}

1. The gait of the patient did bear a relationship to the angles measured, but angles alone were not sufficient to predict the result of surgery. The gait might be good if the patient had a poor range of movement provided the resting angle of the foot was fairly high. It could also be good with a lower resting angle but a better range of movement.

2. The resting angle did not seem to be significantly better in any one technique. It dropped to varying degrees in all techniques.

3. Patients were happier if the operation gave them a good range of movement. They were not so happy with a foot they could not move even if their gait was apparently good. This could be due to other factors such as difficulty climbing hills or walking on rough ground and further investigations need to be done in this respect.

4. The active range of dorsiflexion was greatest with the single tendon techniques. It was less at least initially in the Carayon two-tendon techniques.

5. There were more cases with inversion problems in the circumtibial single-tendon technique. Some of these cases had a definite tendency to inversion preoperatively. It would seem sensible then to avoid this technique where there is a tendency to inversion preoperatively. Results did not however prove that the technique would cause inversion in a foot which preoperatively was straight and mobile.

6. Records were not sufficient to show whether the operations really helped to prevent any further foot damage and ulceration. There were certainly a large proportion of patien ts with postoperative as well as preoperative ulcers. This might have been because such patients were more likely to return to the hospital and be seen for review whereas those with no further problems might not have bothered to come.

7. My impression from the study was that for young patients with a good mobile straight foot preoperatively, the single tendon transfers were preferable as they gave a better range of movement. However for any patients with a tendency to inversion or for those with badly deformed or stiff feet where range of 
movement was less important than stability the two-tendon Carayon techniques might be preferable.

\section{Acknowledgement}

I would like to thank the doctors of ALERT and my head of department Miss Jean Watson for their help and encouragement whilst undertaking this follow-up study. 